\title{
Weight Loss, CTCAE
}

National Cancer Institute

\section{Source}

National Cancer Institute. Weight LoSS, CT CAE. NCI Thesaurus. Code C55339.

A finding characterized by a decrease in overall body weight; for pediatrics, less than the baseline growth curve. 